\title{
Proceeding
}

Supplementary Issue: Winter Conferences of Sports Science. Costa Blanca Sports Science Events, 24 April 2020. Alicante, Spain.

\section{The Orexin-A serum levels are strongly modulated by physical activity intervention in diabetes mellitus patients}

\author{
RITA POLITO ${ }^{1}$, VINCENZO CRISTIAN FRANCAVILLA ${ }^{2}$, ANTONIO AMBROSI ${ }^{3}$, NICOLA TARTAGLIA ${ }^{3}$, \\ DOMENICO TAFURI ${ }^{4}$, MARCELLINO MONDA ${ }^{5}$, ANTONIETTA MESSINA ${ }^{5}$, FRANCESCO SESSA ${ }^{1}$, GIROLAMO DI \\ MAIO $^{5}$, ALBERTO AMETTA ${ }^{6}$, ALESSIA SCARINCI ${ }^{7}$, VINCENZO MONDA ${ }^{5}$, GIOVANNI MESSINA ${ }^{1}$ \\ ${ }^{1}$ Department of Clinical and Experimental Medicine, University of Foggia, Italy \\ 2School of Engineering, Architecture, and Motor Sciences, Kore University of Enna, Enna, Italy \\ ${ }^{3}$ Department of Medical and Surgical Sciences, University of Foggia, Italy \\ ${ }^{4}$ Department of Motor Sciences and Wellness, University of Naples "Parthenope", Naples, Italy \\ ${ }^{5}$ Department of Experimental Medicine, Section of Human Physiology and Unit of Dietetic and Sport Medicine, \\ University of Campania Luigi Vanvitelli, Naples, Italy \\ ${ }^{6}$ Department of Economy, University of Foggia, Italy \\ ${ }^{7}$ Department of Education, Psychology, Communication, University of Bari, Italy
}

\begin{abstract}
The Orexin-A (hypocretin-1) is a neuropeptide secreted by neurons in the lateral hypothalamus. This protein regulates physiological and behavioural processes that have an essential impact on energy balance and metabolic status, physical activity, blood glucose levels, and food intake. Furthermore, that orexin-A regulates insulin sensitivity, energy expenditure and metabolic rate and is involved in immune processes and then regulate inflammatory response, with an anti-inflammatory action. Diabetes mellitus (T2DM) is a worldwide health problem associated with obesity and sedentary lifestyle. High glycaemic levels and lipid serum profile, low col-HDL, or hypertension and increased body mass index (BMI) are significantly associated with increased T2DM risk and with increased cardiovascular mortality and morbidity in T2DM patients. For these reasons the aim of this study is to evaluate the biochemical and anthropometric parameters, orexin-A levels by ELISA test and western blotting analysis, and inflammatory cytokines levels such as TNF-a, IL-8 and IL-10 by ELISA test in subjects affected by diabetes mellitus following an accurate physical activity program at baseline, after 3 months and after 6 months. We found that there is a ameliorate of many anthropometric and biochemical parameters; furthermore, there is a statistical increase of orexin-A serum levels already after 3 months compared to baseline in T2DM subjects and also there is a strongly modulation in inflammatory cytokines expression. These found indicates that the physical activity has beneficial effects not only on anthropometric and biochemical parameters but also on orexin-A levels, and then on CNS.
\end{abstract}

Keywords: Type 2 diabetes mellitus (T2DM); Orexin-A; TNF-a; IL-8; IL-10; Physical activity.

\section{Cite this article as:}

Polito, R., Francavilla, V.C., Ambrosi, A., Tartaglia, N., Tafuri, D., Monda, M., Messina, A., Sessa, F., Di Maio, G., Ametta, A., Scarinci, A., Monda, V., \& Messina, G. (2020). The Orexin-A serum levels are strongly modulated by physical activity intervention in diabetes mellitus patients. Journal of Human Sport and Exercise, 15(2proc), S244-S251. doi:https://doi.org/10.14198/jhse.2020.15.Proc2.15

Corresponding author. Department of Clinical and Experimental Medicine, University of Foggia, Italy.

E-mail: rita.polito@unicampania.it

Supplementary Issue: Winter Conferences of Sports Science. Costa Blanca Sports Science Events, 24 April 2020. Alicante, Spain.

JOURNAL OF HUMAN SPORT \& EXERCISE ISSN 1988-5202

(c) Faculty of Education. University of Alicante

doi:10.14198/jhse.2020.15.Proc2.15

S244 


\section{INTRODUCTION}

Diabetes is a major cause of morbidity and premature mortality in many countries, in particular related to cardiovascular disease. It is associated with obesity and sedentary lifestyle. Type 2 diabetes mellitus (T2DM) is characterized by high glycaemic levels and lipid serum profile, low col-HDL, or hypertension and increased body mass index (BMI) increasing cardiovascular mortality and morbidity in patients affected by diabetes mellitus (Bruton et al, 2016). Physical activity and exercise is known to have a beneficial effect on a variety of physiological and pathophysiological conditions such as diabetes and/or cardiovascular disease, modulating blood pressure, lipid profiles, endothelial function, body composition and insulin sensitivity (Erickson et al, 2011).

For these reasons, exercise is recommended for both the prevention of diabetes and the treatment of people with diabetes. In addition, physical activity improves general health preventing obesity and diseases correlated and reducing cognitive decline associated with age (Julian et al, 2018). It reduces chronic inflammation and oxidative stress through the modulation of many mediators such as pro-inflammatory and anti-inflammatory cytokines and also through the production of Orexin-A (Messina et al 2018).

The Orexin-A (hypocretin-1) is a neuropeptide secreted by neurons in the lateral hypothalamus. This neuropeptide is an important link between peripheral energy balance and the CNS mechanisms (Messina et al, 2017).

This hormone is influenced by various metabolic molecules such as glucose and leptin, acting on energy balance and metabolic status, blood glucose levels, and food intake (Cavaliere et al, 2018; Cavaliere et al, 2019).

Furthermore, that orexin-A regulates insulin sensitivity, energy expenditure and metabolic rate and is involved in immune processes and then regulate inflammatory response, with an anti-inflammatory action. The physical activity has an important act on Orexi-A production (Moscatelli et al, 2016), increasing Orexin-A levels. In the lights of these evidences, the aims of this study is to evaluate the biochemical and anthropometric parameters, Orexin-A serum levels and pro-inflammatory cytokines levels such as TNF-a, IL8 and IL-10 in a cohort of T2DM patients following a physical activity program at baseline and after three and six months.

\section{MATERIAL AND METHODS}

\section{Participants}

Twenty T2DM patients (10 females, 10 males), aged between 50-65 years (mean $57 \pm 4,5$ years), volunteered to participate in the study. Written informed consent for participation in the study was obtained from every patient. This study was performed in accordance with the Declaration of Helsinki and approved by the local ethics committee. Participants were excluded if they had a prior medical history of renal insufficiency, hyperuricemia, severe hepatic insufficiency, atrioventricular block, heart failure, cardiovascular and cerebrovascular diseases, unbalanced hypokalaemia, neoplasms, pregnancy and lactation. The anthropometric and biochemical characteristics of the 20 included patients are evaluated at baseline and after three and six months of physical activity program. Blood tests were taken at baseline and 3 and 6 months, after a $12 \mathrm{~h}$ fast. Fasting blood samples were collected at 8:00 am from an antecubital vein, using a $21 \mathrm{G}$ Vacutainer blood collection set (BD Diagnostics, Franklin Lakes, NJ, USA). Blood samples were centrifuged and the resultant serum stored at $-80^{\circ} \mathrm{C}$, until use. 


\section{Physical activity protocol}

T2DM patients following this mixed exercise program. The protocol consist in 90 minutes per week in 2 session. In the first time T2DM patients following an aerobic session, 20 minutes on treadmill $(55-70 \%$ of VO2max). In the second step the patients are subjected to resistance exercise (65\% of 1RM). The protocol provides transverse thrust movements, frontal traction movements of the upper limbs, distension of the lower limbs, trunk flexions for the abdominals and 3 stretching positions. In addition, the participants underwent echocardiography and cardiopulmonary exercise testing at baseline and 6 months.

\section{Biochemical assay}

As previously described serum orexin-A concentrations were determined by enzyme-linked immunoassay (ELISA), using a commercial kit, according to manufacturers' instructions (Phoenix Pharmaceuticals, USA). Briefly, Sep-Pak C18 columns (Waters, Milford, MA, USA) were utilized to extract orexin-A from T2DM sera, before and after physical activity protocol (Valenzano et al, 2019).

TNF-a, IL-8 and IL-10 serum levels were analysed by enzyme-linked immunosorbent assay (ELISA) using a commercial kit (BD Opt EIA for human TNF-a, IL-8, IL-10). All ELISA-tests were performed in triplicate and the protocols followed were according to the manufacturers' instructions.

In addition, we analysed Orexin-A serum by western blotting. Serum samples from T2 DM participants were quantified for total proteins by Bradford assay (Bio-Rad, Hercules, CA, USA), and $10 \mu \mathrm{g}$ of total protein was loaded on $10 \%$ SDS-PAGE gels. The immunoblots were blotted with Orexin-A Antibody according to manufacturers' instructions (Thermo Fisher USA).

\section{Statistical analysis}

Because this study used a "pre-post" design and the comparison of interest was the change from baseline to 3 and 6 months physical activity intervention, a two-tailed paired t test was used to test for statistical significance of outcome variables. Statistical analyses were performed using the StatView software 5.0.1.0. All data are presented as mean \pm SE. A $p$ value of $\leq .05$ was used for statistical significance.

\section{RESULTS}

\section{Anthropometric and biochemical parameters of T2DM patients before and after physical activity protocol}

Our results show a significant change in the anthropometric and biochemical parameters of T2DM patients before and after physical activity protocol. First of all, anthropometric parameters such as weight and BMl are statistically reduced in T2DM patients before and after physical activity intervention (Table 1). Furthermore, there is a strongly modulation of all biochemical parameters, such as glycaemic and lipid profile. Interestingly, as show ELISA test and western blotting analysis the Orexin-A levels in these patients after physical activity intervention are statistically increased already after three months (Figure 1). In addition, proinflammatory cytokines levels such as TNF-a and IL-8 are strongly reduced after 6 months of physical activity protocol compared to before physical activity intervention. On the contrary IL-10 serum levels are strongly and statistical increased in T2DM patients after physical activity protocol (Figure 2). 
Table 1. Anthropometric and biochemical parameters of T2DM patients before and after physical activity protocol.

\begin{tabular}{lcccc}
\hline & \multicolumn{5}{c}{ T2DM } \\
\hline & T0 & T3 months & T6 months & p-value \\
\hline Age & $57 \pm 4.5$ & & & $\mathrm{~ns}$ \\
BMI $\left(\mathrm{kg} / \mathrm{m}^{2}\right)$ & $29.19 \pm 4.78$ & $27.76 \pm 3.62$ & $25.5 \pm 4.4$ & $<.05$ \\
Glycemia $(\mathrm{mg} / \mathrm{dl})$ & $183 \pm 8.9$ & $128.4 \pm 9.3$ & $99 \pm 7.5$ & $<.05$ \\
Total Cholesterol (mg/dl) & $243 \pm 10$ & $173.91 \pm 9.3$ & $150.3 \pm 7.5$ & $<.05$ \\
HDL $(\mathrm{mg} / \mathrm{dl})$ & $40.13 \pm 9.14$ & $47.76 \pm 9.14$ & $68.2 \pm 3.4$ & $<.05$ \\
LDL $(\mathrm{mg} / \mathrm{dl})$ & $191.83 \pm 6.48$ & $107.57 \pm 7.72$ & $108 \pm 7.72$ & $<.05$ \\
Triglycerides (mg/dl) & $125.54 \pm 5.27$ & $83.25 \pm 26.14$ & $81.45 \pm 2.7$ & $<.05$ \\
Total Bilirubin $(\mathrm{mg} / \mathrm{dl})$ & $0.61 \pm 0.22$ & $0.65 \pm 0.27$ & $0.66 \pm 0.17$ & $\mathrm{~ns}$ \\
Total Protein (g/dl) & $7.8 \pm 0.4$ & $7.13 \pm 0.4$ & $7.01 \pm 0.7$ & $\mathrm{~ns}$ \\
Azotaemia $(\mathrm{mg} / \mathrm{dl})$ & $34.35 \pm 8.43$ & $35 \pm 6.6$ & $35.5 \pm 7$ & $\mathrm{~ns}$ \\
Calcemia $(\mathrm{mg} / \mathrm{dl})$ & $9.57 \pm 0.33$ & $9.72 \pm 0.35$ & $9.65 \pm 0.27$ & $\mathrm{~ns}$ \\
Sodium (mmol/L) & $139.19 \pm 2.48$ & $139.18 \pm 2$ & $138.9 \pm 2.7$ & $\mathrm{~ns}$ \\
\hline
\end{tabular}
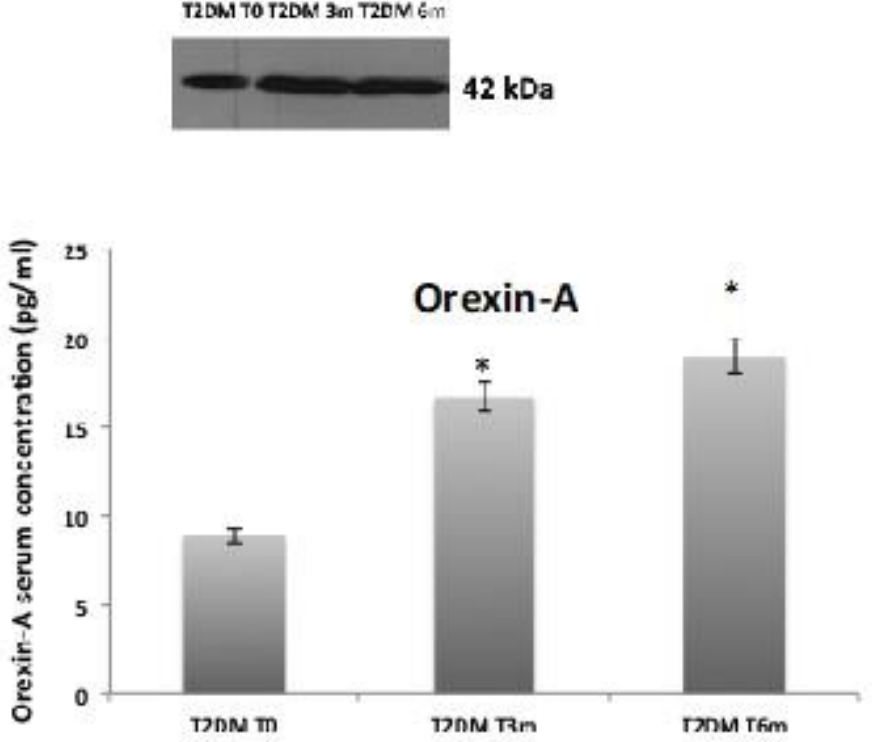

Figure 1. Orexin-A levels strongly modulated by physical activity in T2DM patients. Orexin-A serum levels statistically increased in T2DM patients before and after 3 months and 6 months physical activity protocol. 

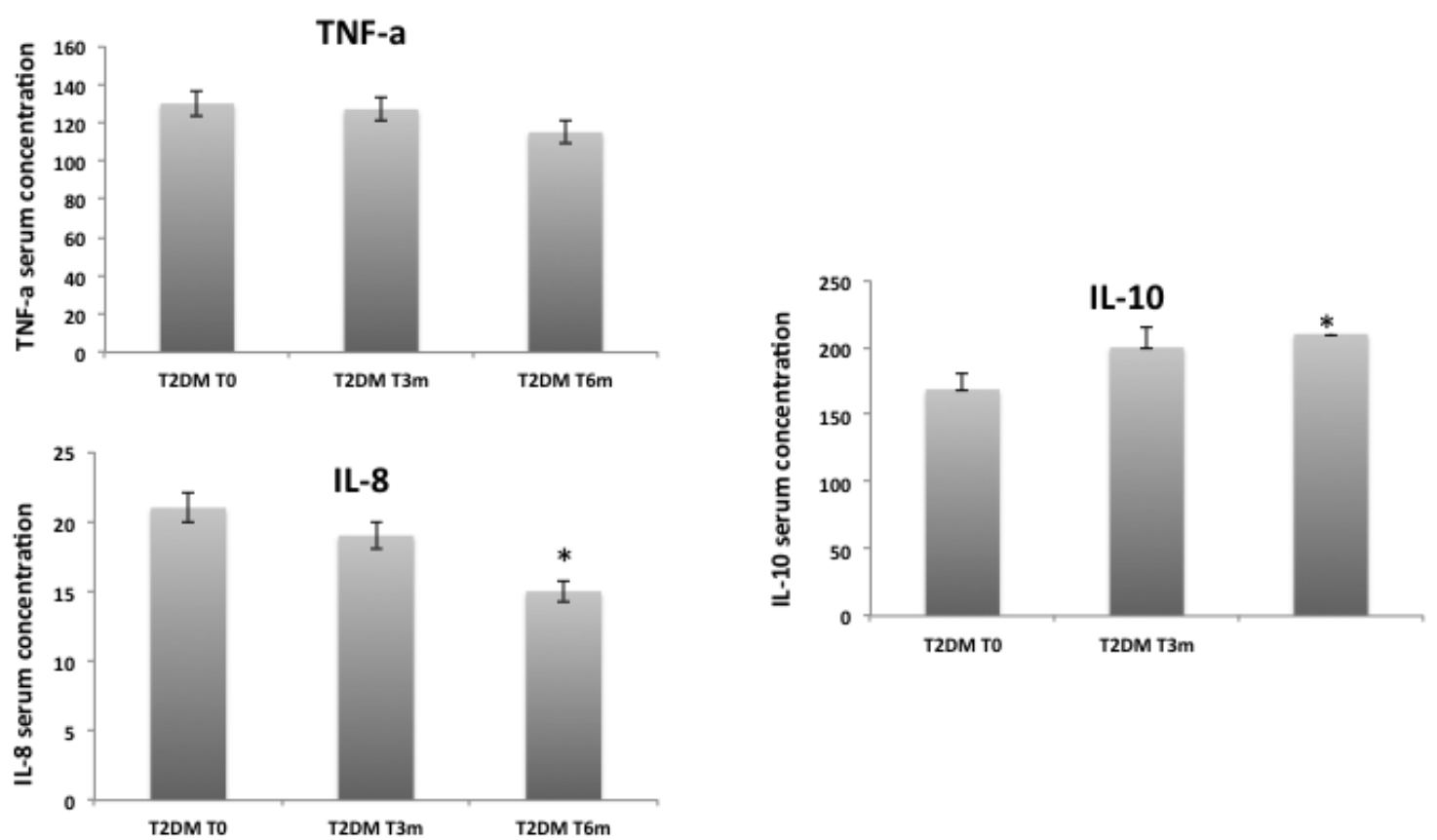

Figure 2. Pro-inflammatory and anti-inflammatory cytokines are modulated by physical activity in T2DM patients. TNF-a and IL-8 are decreased after 3 and 6 months of physical activity protocol; on the contrary IL10 are strongly increased after physical activity protocol.

\section{DISCUSSION}

Type 2 diabetes mellitus is a multifactorial disease characterized by elevated glycaemic serum levels, altered lipid profile and also hypertension. It consists in a mix of genetic and environmental factors (Chatterjee $S$ et al, 2017). This epidemic is associated with increases in obesity and physical inactivity, so exercise would appear to be a key strategy for reversing this trend (Valenzano A et al, 2019). There is ample evidence that physical exercise protects against cardiovascular, bone and joint, and metabolic disorders such as diabetes and that it is an effective means in the prevention and treatment of certain types of cancer (Francavilla $\mathrm{C}$ et al, 2013, Francavilla et al, 2015). It follows than that physical well-being plays a central role in our lives. Physical activity improves cardiovascular performance and protects against many preventable diseases. We can consider the physical activity as a therapy in many metabolic and inflammatory disease and also in obesity and diseases correlated (Francavilla et al, 2017, Patti et al, 2017; Bianco et al, 2018). In this scenario, an important mediator related to obesity development and also physical activity is Orexin-A/Hypocretin 1, a neuropeptide synthesized in the lateral hypothalamus (Messina et al, 2016). It plays an important role in the regulation of appetite: indeed, a reduction in the amount of Orexin- A determines a reduction of appetite (Sperandeo et al, 2018). In addition, orexin-A regulates insulin sensitivity, energy expenditure and metabolic rate and is involved in immune processes and then regulate inflammatory response, with an anti-inflammatory action. The production of orexin-A by correct nutrition and by physical activity, has an important role in prevention of metabolic and inflammatory disease and also of the aging (Monda et al, 2019; Giuditta et al, 2013). In the light of these evidences, in this study we evaluated Orexin-A serum levels and cytokines serum levels in a cohort of patients affected by type 2 diabetes mellitus. We found an increase of Orexin-A levels and IL-10 which are anti-inflammatory mediators and also we found a reduction in TNF-a and IL-8 serum levels which are pro-inflammatory mediators. Several studies supported our findings, indeed Kotz et al. 
reported that Orexin-A is increased by physical activity (Kotz et al, 2006; Eyman et al, 2013). In addition, the plasma Orexin-A level was associated with physical activity in obese and overweight people, including many aspects of daily life, such as working, domestic work, and especially walking. A high Orexin-A level was related to moderately active movement in living habits (Kotz et al. 2006; Moscatelli et al, 2015; Messina et al, 2015). The higher Orexin-A tone is an endogenous factor that predicts physical activity, improving BMI and the energy expenditure aspect (Mazzeo et al, 2013; Viggiano et al, 2008; Sessa et al, 2018). In addition, literature data suggest that Orexin-A may be a potential therapeutic method for controlling obesity by regulating physical activity and energy expenditure. Furthermore, the beneficial effects of physical activity are not only on metabolic rate but also on cognitive aspects. Indeed, it improves cognitive performance also through the production of Orexin-A. (Colcombe et al, 2004; Erickson et al, 2011; Lindwall et al, 2008). In addition, the ameliorates of all biochemical parameters and in particular, of glycemia in these T2DM patients is also attributable to Orexin-A serum levels increased. Indeed, Gonzalez et al, 2012, reported that orexin neurons act as adaptive glucose sensors and are inhibited directly at higher glucose concentrations, suggesting that hyperglycaemia results in decreased orexin signalling (Burdakov et al, 2006; González et al, 2012; Williams et al, 2008). Another important beneficial effect of physical activity is to reduce proinflammatory response, as support by Ziegler et al, physical activity is able to reduce pro-inflammatory cytokines production, acting on sirtuine pathways and also reducing oxidative stress (Ziegler et al 2015). For these reasons, in T2DM patients physical activity protocol has beneficial effects not only on metabolic status but also on the inflammatory status, also reducing the risk of development correlated diseases to inflammatory state.

\section{CONCLUSION}

Physical activity may improve health status, it has an important role in the prevention and in the treatment of T2DM and its associated diseases and reducing cognitive decline associated with age. Therapies aimed at increasing physical activity would contribute to better clinical outcomes in the treatment of T2DM, obesity and metabolic syndrome, high-incidence diseases in developed countries (Perez-Leinghton et al, 2012). Our study show that these improvements also involved Orexin-A response and inflammatory cytokines. In conclusion, the increase of Orexin-A and IL-10 and the decrease of TNF-a and IL-8 concentrations corresponds to the improvement of wellness and may be used for controlling T2DM by regulating physical activity and energy expenditure.

\section{REFERENCES}

Bianco A, Gentile A, Boca S, et al. (2018). An exploratory analysis of factors associated with healthrelated physical fitness in adolescents. Sustainability; 10:1847. https://doi.org/10.3390/su10061847

Brunton S et al. (2016). Pathophysiology of Type 2 Diabetes: The Evolution of Our Understanding. J Fam Pract.

Burdakov, D., Jensen, L. T., Alexopoulos, H., Williams, R. H., Fearon, I. M., O'Kelly, I., et al. (2006). Tandem-Pore K+ Channels Mediate Inhibition of Orexin Neurons by Glucose. Neuron. https://doi.org/10.1016/j.neuron.2006.04.032

Cavaliere G, Trinchese G, Penna E, Cimmino F, Pirozzi C, Lama A, Annunziata C, Catapano A, Mattace Raso G, Meli R, Monda M, Messina G, Zammit C, Crispino M, Mollica MP. (2019). High-Fat Diet Induces Neuroinflammation and Mitochondrial Impairment in Mice Cerebral Cortex and Synaptic Fraction. Front Cell Neurosci.;13:509. https://doi.org/10.3389/fncel.2019.00509

Cavaliere G, Viggiano E, Trinchese G, De Filippo C, Messina A, Monda V, Valenzano A, Cincione RI, Zammit C, Cimmino F, Catapano A, Sessa F, Messina G, Monda M, Crispino M, Mollica MP. (2018). 
Long Feeding High-Fat Diet Induces Hypothalamic Oxidative Stress and Inflammation, and Prolonged Hypothalamic AMPK Activation in Rat Animal Model. Front Physiol. 9:818. https://doi.org/10.3389/fphys.2018.00818

Chatterjee $S$ et al. (2017). Type 2 diabetes. Lancet.

Colcombe SJ, Kramer AF, Erickson KI, Scalf P, McAuley E, Cohen NJ, et al. (2004). Cardiovascular fitness, cortical plasticity, and aging. Proc. Natl. Acad. Sci. U. S. A. https://doi.org/10.1073/pnas.0400266101

Cristian V Francavilla, Francesco Sessa, Monica Salerno, Giuseppe D Albano, Ines Villano, et al. (2018). Influence of Football on Physiological Cardiac Indexes in Professional and Young Athletes. Front Physiol.; 9:153. https://doi.org/10.3389/fphys.2018.00153

Erickson KI, Voss MW, Prakash RS, Basak C, Szabo A, Chaddock L, et al. (2011). Exercise training increases size of hippocampus and improves memory. Proc. Natl. Acad. Sci. U. S. A. https://doi.org/10.1073/pnas.1015950108

Francavilla G, Francavilla C. (2013). Physical exercise is therapy. MED SPORT;66:625-8.

Francavilla VC, Bongiovanni T, Genovesi F, Minafra P, Francavilla G. (2015). Localized bioelectrical impedance analysis: how useful is it in the follow-up of muscle injury? A case report. MED SPORT; 68:323-34.

Francavilla VC, Bongiovanni T, Todaro L, Di Pietro V, Francavilla G. (2017). Probiotic supplements and athletic performance: a review of the literature. MED SPORT; 70:000-000 https://doi.org/10.23736/S0025-7826.17.03037-X

Giuditta A, Chun JT, Eyman M, Cefaliello C, Bruno AP and Crispino M. (2008). Local gene expression in axons and nerve endings: the glia-neuron unit. Physiol. Rev. 2008. 88:515-555. https://doi.org/10.1152/physrev.00051.2006

Eyman M, Cefaliello C, Mandile P, Piscopo S, Crispino M, Giuditta A. (2013). Training old rats selectively modulates synaptosomal protein synthesis. J. Neurosci. Res. 2013. 91:20-29. https://doi.org/10.1002/jnr.23133

González JA, Jensen LT, Fugger L, and Burdakov D. (2012). Convergent inputs from electrically and topographically distinct orexin cells to locus coeruleus and ventral tegmental area. Eur. J. Neurosci. https://doi.org/10.1111/j.1460-9568.2012.08057.x

Julian V, Thivel D, Costes F, Touron J, Boirie Y, Pereira B, Perrault H, Duclos M, Richard R. (2018). Eccentric Training Improves Body Composition by Inducing Mechanical and Metabolic Adaptations: A Promising Approach for Overweight and Obese Individuals. Front. Physiol. https://doi.org/10.3389/fphys.2018.01013

Kotz CM, Wang C, Teske JA, Thorpe A J, Novak CM, Kiwaki K, et al. (2006). Orexin A mediation of time spent moving in rats: Neural mechanisms. Neuroscience. https://doi.org/10.1016/..neuroscience.2006.05.028

Lindwall M, Rennemark M, and Berggren T. (2008). Movement in mind: The relationship of exercise with cognitive status for older adults in the Swedish National Study on Aging and Care (SNAC). Aging Ment. Heal. https://doi.org/10.1080/13607860701797232

Mazzeo F, Motti ML, Messina G, et al. (2013). Use of nutritional supplements among south Italian students of Physical Training and Sport University. Current Topics in Toxicology. 9:21-26.

Messina A, Bitetti I, Precenzano F, lacono D, Messina G, Roccella M, Parisi. et al. (2018). Non-Rapid Eye Movement Sleep Parasomnias and Migraine: A Role of Orexinergic Projections. Front Neurol.;9:95. https://doi.org/10.3389/fneur.2018.00095

Messina A, Monda V, et al. (2017). Role of the orexin system on arousal, attention, feeding behaviour and sleep disorders. Acta Medica Mediterr., 33(4):645-64. https://doi.org/10.19193/0393$\underline{6384 \_2017 \_4 \_096}$ 
Messina G, Zannella C, Monda V, Dato A, Liccardo D, De Blasio S, et al. (2015). The Beneficial Effects of Coffee in Human Nutrition. Biol Med.

Messina A, De Fusco C, Monda V, Esposito M, Moscatelli F, Valenzano A, et al. (2016). Role of the orexin system on the hypothalamus-pituitary-thyroid axis. Front. Neural Circuits. 10. https://doi.org/10.3389/fncir.2016.00066

Monda V, Villano I, Messina A, Valenzano A, Salerno M, Signorelli S, et al. (2019). Aerobic exercise and orexin A: Role of sympathetic activity and redox system. J Biol Reg and Hom Ag. 33, 587-592.

Moscatelli F, Messina G, Valenzano A, Monda V, Viggiano A, Messina A, Petito A, Triggiani Al, Ciliberti MA, Monda M, Capranica L, Cibelli G. (2016). Differences in corticospinal system activity and reaction response between karate athletes and non-athletes. Neurological Sciences. 37(12). https://doi.org/10.1007/s10072-016-2693-8

Moscatelli F, Messina G, Valenzano A, Monda M, et al. (2015). Relationship between RPE and Blood Lactate after Fatiguing Handgrip Exercise in Taekwondo and Sedentary Subjects. Biol Med. https://doi.org/10.4172/0974-8369.1000s3008

Patti A, Bianco A, Karsten B, Montalto MA, Battaglia G, et al. (2017). The effects of physical training without equipment on pain perception and balance in the elderly: A randomized controlled trial. Work. 57:23-30. https://doi.org/10.3233/wor-172539

Sessa F, Anna V, Messina G, Cibelli G, Monda V, Marsala G, Ruberto M, Biondi A, Cascio O, Bertozzi G, Pisanelli D, Maglietta F, Messina A, Mollica MP, Salerno M. (2018). Heart rate variability as predictive factor for sudden cardiac death. Aging. 23;10(2):166-177. https://doi.org/10.18632/aging.101386

Sperandeo R, Maldonato MN, Messina A, Cozzolino P, Monda M, Cerroni F, et al. (2018). Orexin system: Network multi-tasking. Acta Medica Mediterr. 34, 349-356. https://doi.org/10.19193/0393$6384 \_2018 \_2 \quad 55$

Valenzano A, Polito R, Trimigno V, Di Palma A, Moscatelli F, Corso G, Sessa F, Salerno M, Montana A, Di Nunno N, Astuto M, Daniele A, Carotenuto M, Messina G, Cibelli G, Monda V. (2019). Effects of Very Low Calorie Ketogenic Diet on the Orexinergic System, Visceral Adipose Tissue, and ROS Production. Antioxidants 2019. 8(12). https://doi.org/10.3390/antiox8120643

Viggiano A, Vicidomini C, Monda M, Carleo D, Carleo R, Messina G, Viggiano A, Viggiano E, De Luca B. (2009). Fast and low-cost analysis of heart rate variability reveals vegetative alterations in noncomplicated diabetic patients. J Diabetes Complications. 23(2):119-23. https://doi.org/10.1016/j.jdiacomp.2007.11.009

Viggiano E, Monda V, Messina A, Moscatelli F, Valenzano A, Tafuri D, Cibelli G, De Luca B, Messina G, Monda M. (2016). Cortical spreading depression produces a neuroprotective effect activating mitochondrial uncoupling protein-5. Neuropsychiatr Dis Treat. 11;12:1705-10. https://doi.org/10.2147/ndt.s107074

Williams RH, Alexopoulos H, Jensen LT, Fugger L, Burdakov D. (2008). Adaptive sugar sensors in hypothalamic feeding circuits. Proc Natl Acad Sci U. S. A. https://doi.org/10.1073/pnas.0802687105

Ziegler DR, Ribeiro LC, Hagenn M, et al. (2003). Ketogenic diet increases glutathione peroxidase activity in rat hippocampus. Neurochem Res. 28: 1793-1797.

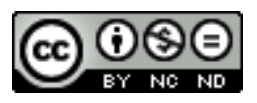

This work is licensed under a Attribution-NonCommercial-NoDerivatives 4.0 International (CC BY-NC-ND 4.0). 